\title{
La traduction de l'implicite dans \\ Ténèbres à midi de Théo Ananissoh
}

Samir Ismaïl M. Ismaïl ${ }^{(*)}$

\section{Introduction}

Une traduction ne concerne pas seulement un passage entre deux langues, mais entre deux cultures, ou deux communautés. Ce passage indique non seulement que le destinataire change mais avec lui changent aussi les connaissances du monde. Le nouveau lecteur n'a pas forcement le même point de départ que le lecteur d'origine. Cela veut dire que le traducteur s'efforce de se distancer dans sa propre langue en utilisant le même niveau utilisé par l'auteur principal. Il tient compte des règles linguistiques, mais aussi d'éléments culturels, au sens le plus large du terme. De plus, le traducteur devient un coauteur ou re-écrivain, dont le travail connaît les mêmes difficultés que l'auteur d'origine.

Parmi ces difficultés, le traducteur envisage le problème de l'implicite. Ce dernier est défini comme un contenu présent dans le texte-source sans être directement

(*) Maître de langue à la Faculté des Lettres, Département de FrançaisUniversité de Sohag.

Cette recherche fait une partie d'une thèse de Magistère, intitulée: "Ténèbres à midi de Théo Ananissoh Traduction en arabe suivie d'une étude des problèmes de traduction", Sous la direction du. Prof. Oussama Mohammed Nabil ALI, Faculté de Langues et de Traduction, Université d'AL-AZHAR. \& du Dr. Hanan Abo Eltaher Ebaid, Faculté des Lettres, Université de Sohag. 
exprimé. Il désigne tout ce qui se trouve dans le discours sans être littéralement prononcé. Il s'agit du sens sousentendu de tout ce qui n'est pas directement exprimé. Il n'est pas facile de traduire l'implicite dans un texte littéraire puisqu'il est considéré comme un acte indirect du langage.

Le but de cette recherche, c'est de savoir pourquoi les auteurs francophones écrivent en langue étrangère. Nous allons évoquer des messages non exprimés au cour du corpus et découvrir des réalités qui se cachent derrière les mots. Nous allons interpréter les effets produits par le langage gestuel ou mimique au quel recourt l'auteur. Nous allons profiter aussi des gestes, des actions verbales et nonverbales, des non-dits et d'autant d'autres manifestations pour transférer directement le message du texte source au texte cible.

\section{La problématique de la recherche}

Avant de commencer à traduire un texte, il faut le comprendre complétement. Cette compréhension permet de faire intervenir des connaissances aussi bien linguistiques qu'extralinguistiques. Elle joue un rôle essentiel dans le processus de traduction. Selon le Robert et M. Lederer : «Comprendre un texte, c'est faire appel à une compétence linguistique et, simultanément, à un savoir 
encyclopédique afin de faire correspondre à quelque chose le contenu véhiculé par le texte lui-même.»"

En effet, le traducteur est avant tout un lecteur. Mais c'est un lecteur particulier car il lui est nécessaire de lire analytiquement le texte original pour comprendre ce dont il s'agit. Il doit décrypter l'intention et le vouloir-dire de l'auteur pour obtenir une traduction logique, identique et fluide à la lecture. Il doit mettre en considération qu'il ne traduit pas pour comprendre un texte mais pour le faire comprendre aux autres lecteurs qui n'ont pas accès direct au texte source. Selon Darbelnet : «La traduction a pour but de faire connaître à d'autres ce qui a été dit ou écrit dans la langue étrangère. Celui qui traduit ne traduit pas alors pour comprendre mais pour faire comprendre. Il a compris avant de traduire.» ${ }^{2}$

Nous sommes alors d'accord pour dire que la traduction dépend en grande partie du sens du message puisqu'elle n'est pas un travail sur la langue, ni sur les mots, mais c'est un travail sur le message et sur le sens. Nous devons alors faire passer les éléments représentés directement et indirectement dans l'œuvre à traduire. En d'autres termes, tout texte est pourvu d'un sens explicite et d'un sens implicite. L'explicite est la partie du message qui est réellement exprimée alors que l'implicite représente

\footnotetext{
$\left.{ }^{1}\right)$ Hong- Van D : La théorie du sens et la traduction des facteurs culturels, Synergies Pays riverains du Mékong n 1 - 2010 pp. 141-171

2) Vinay J.P \& Darbelnet J : Stylistique comparée du français et de l'anglais. Méthode de traduction, Paris, Didier, 1977, P. 24
} 
tout ce qui dépasse le signifiant d'un message, le sens littéral d'un énoncé. Selon Chiali : «On nomme sens implicite tout sens qui n'est pas directement lié au signifiant d'un message, mais qui est anticipé, prémédité, à partir des signifiés normalement associés de ce message.»"

L'implicite est une notion commune en littérature du fait de la polysémie des textes littéraires. Cette notion a été étudiée avec clairvoyance par le linguiste Catherine Kerbrat. En bref, les notions d'implicite et de connotation, dans toute forme de discours quelle que soit sa nature, désignent ce qui n'est pas ouvertement et étroitement dit, mais ce qui est plutôt suggéré à penser. D'après Kerbrat, l'implicite est « une chose dite à mots couverts, une arrièrepensée sous entendue entre les lignes». ${ }^{2}$

L'implicite n'est pas donc le contenu principal de l'énoncé. Il s'agit d'idées supplémentaires venant de la signification explicite. Dominique Maingueneau met en évidence cette notion en affirmant qu' : " on peut tirer d'un énoncé des contenus qui ne constituent pas en principe l'objet véritable de l'énonciation mais qui apparaissent à travers les contenus explicites. C'est le domaine de l'implicite»" ${ }^{3}$.

Kerbrat propose deux notions très importantes : le présupposé qui se définit comme un type d'implicite

\footnotetext{
$\left.{ }^{1}\right)$ Lalaoui-Chial F. Z. : Guide de sémiotique appliquée, Oran, OPU, 2008, P.123.

$\left.{ }^{2}\right)$ Catherine K. O. : L'implicite, Paris, Armand Colin, $2^{\mathrm{e}}$ Éd. 1986, P.6

3) Mainngueneau D : Les termes clés de l'analyse du discours, Paris, Seuil, coll. «mé-mo, Lettres », 1996, p.47.
} 
produit par le message linguistique lui-même, et le sousentendu comme un autre type d'implicite produit plus directement en rapport avec les données situationnelles et les activités d'interprétation des interlocuteurs. En d'autres termes, les présupposés sont des informations présentées comme celles qui vont de soi et interprétées en se référant au seul contenu linguistique. Les sous-entendus sont interprétés en se référant au contexte et à l'environnement discursif de leur production.

De tout ce qui précède, nous remarquons que certains locuteurs ne parlent pas directement et choisissent de laisser entendre ou faire entendre, à leurs interlocuteurs ce qu'ils ne veulent pas ou n'osent pas dire explicitement. En effet, nous avons besoin de dire certaines choses comme si nous ne les avions pas dites. $L$ 'implicite est un acte visant à éviter la responsabilité d'avoir dit une chose embarrassante ou interdite. Parfois, la formulation implicite est plus efficace mais elle est toujours plus risquée que la formulation explicite.

Sur le plan pratique, le recours de l'auteur lui-même au Français comme une langue étrangère représente une sorte d'implicite. L'auteur a besoin de dire certaines choses et d'envoyer indirectement un message à l'Europe et à l'Occident. C'est parce que le milieu où il vit ne favorise pas l'écriture en langue maternelle. L'auteur voudrait transmettre un message aux lecteurs par la description des lieux, des éléments de la nature, du régime en place et du milieu où vivent les gens de son temps. 
Durant la colonisation, écrire en langues africaines est perçu comme un acte de résistance. En effet, les premiers écrivains africains n'ont rédigé leurs ouvres qu'en Français pour que ces ouvres soient lues. Ils ont considéré le Français comme la langue de communication par excellence. Ainsi, Senghor répond à une question concernant son emploi de la langue française comme langue d'écriture : «Parce que nous sommes des métis culturels, parce que, si nous sentons en nègres, nous écrivons en français, parce que le français est une langue à vocation universelle, que notre message s'adresse aussi aux Français de France et aux autres hommes, parce que le français est une langue de gentillesse et d'honnêteté»».

Certes, l'écriture dans la langue de l'Autre est un moyen d'expression et d'ouverture sur le monde, mais écrire dans une langue étrangère peut être révélateur de conflits et de maux intérieurs propres à l'auteur lui-même. Ananissoh n'a pas d'autres choix ; il a besoin d'écrire en langue étrangère pour pouvoir décrire ce qui se passe en réalité. Il devrait faire attention. La relation conflictuelle avec le langage se traduit dans son ouvre par une syntaxe volontairement faible, pleine de silence, d'hésitation, de mal- compréhension et des répétitions. Considérons ce passage :

1) Senghor L. S.:Ethiopiques. Paris, Seuil, 1956, P.12 
«C'est aussi un lieu, je l'avoue, qu'il m'est arrivé maintes fois d'imaginer attaqué, détruit et tous ses occupants sans exception tués». ${ }^{1}$

C'est une phrase complexe et d'une syntaxe faible. Elle est composée de plusieurs verbes employés comme adjectifs et d'une proposition incidente. Nous avons à peine compris cette phrase puisqu'elle appartient plus à l'oral qu'à l'écrit. Lors de la traduction, nous n'avons pas pensé au sens littéral mais au vouloir- dire de l'auteur. Ce dernier voudrait dire: ( j'avoue qu'il m'arrive maintes fois d'imaginer d'attaquer cet endroit, de détruire cette construction et de tuer toutes les personnes qui y travaillent sans laisser la vie à personne.) C'est à partir de cette interprétation que va commencer notre choix de traduction. Nous avons alors raffiné le faible énoncé de l'auteur en revenant au sens contextuel pour parvenir enfin à une traduction conforme au vouloir-dire de l'auteur. Ainsi, nous pouvons traduire l'énoncé ci-dessus par :

$$
\begin{aligned}
& \text { كما أنه (قصر الرئاسة) مكان كثيراً ما كان يجمح خيالي إلى اقتحامه } \\
& \text { ودك أسواره وإزهاق أرواح كل شاغليه دون الإبقاء على أحدهم. }
\end{aligned}
$$

Cette traduction nous semble plus pertinente car elle exprime, à nos yeux, les impressions de l'auteur à l'égard d'un lieu imposant. Cet énoncé met aussi l'accent sur une autre vérité ; c'est la mauvaise intention du locuteur à l'égard de cet endroit. Ce qui nous entraîne à poser cette question : pourquoi le locuteur attaque-t-il ce lieu ? C'est

\footnotetext{
1) Ananissoh T. : Ténèbres à Midi, Gallimard,Paris, 2010, P. 22
} 
peut-être parce que l'auteur ne vit pas dans ce pays. Il a alors une mauvaise attitude contre ceux qui s'installent dans son pays et ceux qui le dirigent à partir de la présidence. Cela est certain car l'auteur nous avoue déjà : «Nadine m'est indispensable pour reprendre pied dans un pays qui est plus le sien que le mien». ${ }^{1}$

De même, ce dernier extrait ci-dessus contient une idée implicitement exprimée. L'auteur se sent étranger dans son pays natal. Il passe quelques jours dans une patrie qui ne lui appartient plus. Ce pays appartient à Nadine et non pas à l'auteur lui- même qui y est né. L'auteur mène alors sa vie hors son pays. Il revient étranger pour y décrire quelques lieux et quelques réalités. En analysant l'énoncé ci-dessus et en mettant en considération le sous-entendu, nous pouvons proposer la traduction suivante :

وغناً لي. نادين أكثر أهمية لي حتى تطأ قدمي بلدة باتت وطناً لها أكثر من كونها

Ce qui mérite d'être noté dans cette traduction, c'est le terme pays qui devrait être traduit par بولة بلدة est employé ici au sens de patrie. Nous l'avons traduit alors par وطن, puisqu'il s'agit ici d'une appartenance. Nous sommes alors devant un auteur sans pays ni identité. Il vit dans un pays qui ne lui appartient plus. Il écrit aussi dans une langue étrangère. Il se retrouve alors dans un non-lieu de pensée. Mais, plus tard, l'auteur comprendra qu'il aime profondément son pays.

1) Ibid. P. 15 
De même, le titre de ce récit : Ténèbres à midi, présuppose l'existence d'une idée implicite soulignée par cette question : les ténèbres se voient-elles le jour ou la nuit ? En réalité, ce titre est une expression évocatrice car la notion de ténèbres suscite une interrogation chez le lecteur : y a-t-il vraiment des ténèbres à midi ? Lesquelles ? L'auteur a choisi un titre évocateur, suggestif, qui génère aussi bien l'activité mentale qu'émotionnelle du lecteur afin qu'il cherche et vérifie les présupposés porteurs et identificateurs de cette notion de ténèbres. Cette notion ne désigne que les ténèbres de l'Afrique. Cette dernière est déchirée par des régimes coloniaux et militaires, mais aussi par l'ignorance et la misère qui frappent tout le continent ظلمات في noir. Nous avons traduit littéralement ce titre par pour mettre le nouveau lecteur dans la même situation et pour lui faire penser à un titre cible évocateur et significatif.

Quelquefois, nous trouvons dans le corpus que la parole disparaît complètement pour laisser place au geste, au para-verbale, au-delà du langage propre à l'être humain. Cela nous met aussi devant un véritable défi lors de la traduction de ce langage gestuel. Nous nous apercevons que le texte source est riche d'expressions gestuelles. Ces dernières servent à exprimer implicitement des sentiments, des intentions ou des idées cachés derrière ces gestes. Dans un style à la fois explicite et implicite, l'écrivain togolais sonde la conscience des hommes en temps 
de dictature à travers ce récit haletant et attachant. Observons l'extrait suivant :

"Il secoue la tête, considère la cour d'un air profondément exaspère, revient à moi." Tout est sombre et vide ici. (Il regarde Nadine.) Tu as raison. Je ne supporte plus d'être au milieu d'eux. ('̀ moi). Bestia prend plaisir à assister à l'agonie de ceux qu'il empoisonne....". ${ }^{1}$

La citation ci-dessus met en évidence la façon dont parle l'auteur narrateur avec son interlocuteur, ainsi que les gestes que l'auteur emploie pour exprimer ses idées. L'énoncé il secoue la tête pose un problème de traduction. Il propose deux choix de traduction. Nous pouvons le prendre au sens négatif de refuser ou au sens positif d'accepter volontairement quelque chose. Par recours au contexte, nous constatons qu'il s'agit du sens positif d'insister c'est-àdire d'accepter. Comment peut-on exprimer ce sens en transférant cet énoncé en langue cible ? Pour rendre cet énoncé compréhensible chez le lecteur arabe, nous avons ajouté des mots explicatifs pour rendre explicite le sens implicite d'un énoncé. Nous pouvons décrypter l'énoncé par (وهز رأسه مؤكدا) . L'énoncé cible هز رأسه indique plusieurs

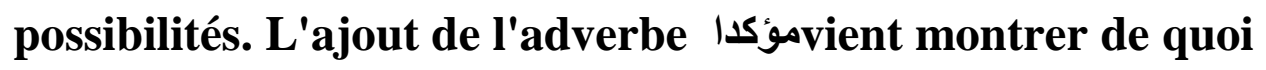
il s'agit.

Quant à l'énoncé (il) revient à moi, il s'agit implicitement de s'adresser à moi c'est-à-dire qu'il poursuit en me disant. Pour traduire l'implicite dans cet énoncé,

1) Ibid. P. 68 
nous avons employé l'énoncé arabe (وتابع قائلا). Cependant, le mot arabe (قائلا) n'est pas exprimé dans la traduction ; il est compris implicitement en langue cible. Nous finissons finit par interpréter l'énoncé par ثاد إلىً وتثابع. Par l'interprétation de l'implicite, nous visons à aider le nouveau lecteur à bien comprendre ce qui est rendu implicite par l'auteur.

Pour l'énoncé mis entre parenthèses (il regarde Nadine), il indique que le locuteur se détourne vers un autre pour s'adresser à lui. Ce geste nous semble très significatif car il nous met au courant de la situation contextuelle. Il nous permet de savoir ce qui devrait être inavoué. Par tels gestes, l'auteur nous mène à bien comprendre les actes de langage répétés dans son texte, ce qui rend simple l'interprétation du texte. Dans le passage vers la langue cible, nous ne faisons qu'insérer cet énoncé entre parenthèses dans le texte cible pour dire : والتقت إلى نادين.

C'est pareil pour l'énoncé (À moi), qui souligne le changement du récepteur. Par (à moi), nous comprenons que l'interlocuteur s'adresse alors au narrateur qui raconte ce récit. Cet énoncé est abrégé. En effet, la forme abrégée des énoncés constitue un type d'implicite. Il s'agit d'une expression elliptique. En interprétant, il nous semble obligatoire de penser au sens complet. Dans cet échantillon

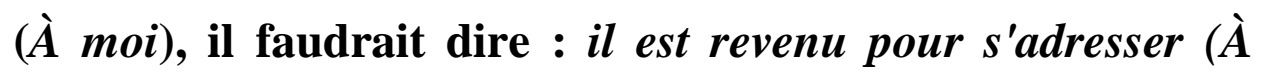
moi). Cet énoncé s'est interprété par ثم استذار ناحيتي وتابع. 
D'ailleurs, le passage ci-dessus nous transmet implicitement un message très important. Ce message est contenu dans l'adverbe de lieu ici et dans le pronom personnel tonique eux. Selon le contexte, nous pourrions prendre l'adverbe ici au sens direct puisque le narrateur est assis au café appelé le 63. Mais, il faudrait penser au sens implicite puisqu'il s'agit du pays natal. L'auteur voudrait dire que tout est sombre et vide dans ces pays africains. Le pronom eux qui ne renvoie pas à de personnes précises fait allusion à ceux qui gouvernent le pays. D'ailleurs l'interlocuteur avoue qu'il ne supporte plus vivre au milieu de ceux qui exercent le pouvoir dans ce pays. Ces gens-là sont méchants et injustes. Tout le régime est alors militaire. C'est la vérité effroyable dont on a froid dans le dos et dont l'auteur parle implicitement. L'énoncé peut avoir alors un signifié explicite qui dissimule un autre signifié implicite. C'est le sens implicite qui constitue le propos véritable de l'auteur.

En transformant ce message, nous étions autant que possible fidèles au texte source. Notre but n'est pas de rendre explicite tous les sens implicites dans le texte, mais d'aider le nouveau lecteur à appréhender le sens et l'effet voulu par l'auteur du texte original. Nous nous efforçons de mettre le lecteur dans des situations d'arrivée semblables. C'est donc à ce dernier de saisir le sens et de comprendre les intentions du locuteur à travers une traduction plus claire et plus proche de l'original. Le rôle du traducteur consiste alors à interpréter ce qui est nécessaire pour la 
compréhension du message. Nous nous sommes contenté de traduire l'adverbe ici par هistout en laissant au lecteur la chance de deviner le sens implicite appartenant au pays tout entier ; le pronom personnel eux, déjà répété par ils, s'est traduit par هؤلاء; ce pronom démonstratif arabe désigne chez le lecteur arabe le même message voulu par l'auteur.

De surcrôit, le lieu où se passe le discours ci-dessus révèle une autre idée très importante. C'est le numéro 63 qui désigne le nom d'un café. L'auteur lui-même nous a montré le sens implicite de ce nom : c'est au mois de janvier 1963, lors du coup d'Etat que Sylvanus Olympio a été assassiné. Ce dernier devrait être le premier président de la République togolaise. C'est à partir de cette date que le territoire togolais est tombé sous un régime militaropolicier et remis dans le bain franco-africain. L'assassinat d'un premier président africain élu a signé ainsi le début de la fin de la marche du peuple africain vers son indépendance.

Tout ce qui précède n'est pas exprimé directement dans le texte source mais il est compris implicitement par le retour à l'histoire politique du Togo et du continent africain. L'auteur voudrait alors susciter la conscience de l'humanité en temps de dictature à travers un récit touchant et horrible. Il recourt à l'implicite afin d'éviter certaines réactions inattendues, non souhaitées. Il préfère faire entendre ou laisser entendre plutôt que de parler ouvertement. Il cherche un langage de dissimulation, de 
feinte, de déguisement. Il a besoin d'un langage particulier qui exprime ses véritables intentions. C'est pour cela qu'il se sert de l'implicite. En revenant à la citation ci-dessus, on finit par la traduire comme ainsi :

وهز رأسه مؤكداً، وأخذ يتأمل الباحة بحالة تفيض حزنا، ثم عاد إلىَ، وتابع:

$$
\begin{aligned}
& \text { - كل شيء هنا بات مظلماً وشاغراً. } \\
& \text { والتفت إلى نادين: }
\end{aligned}
$$

- أَصبتِ كبد الحقيقة، فلم اعد صابراً على البقاء بين هؤلاء.

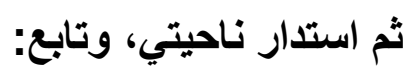

إن بيستيا يستمتع بروئية احتضار هؤلاء الذين يسقيهم السم.

En outre, l'auteur confie une tâche difficile à son narrateur, celle de rendre l'expression implicite presque visible. Ce narrateur est attentif aux présupposés, aux allusions, aux insinuations et aux sous-entendus que comportent les énoncés des personnages. Il paraît aussi très intéressé aux autres moyens d'expression, tels que les expressions faciales, en vue de vérifier la sincérité de l'expression verbale. La construction de la mimique comme unité discursive appartient au discours implicite. Notons ce passage :

«Il dit cela d'un air calme, presque indiffèrent. Il se masse le visage ensuite, pose les coudes sur la table- se rapprochant ainsi de moi [...] Il plisse le front: C'était clair pour elle comme pour moi : ou l'humiliation ou la mort. Parler, discuter, protester est tout à fait futile». ${ }^{1}$

1) Ibid. P. 60-61 
Cet exemple nous montre le rôle de la mimique. Celle-ci sert à rendre explicite les actes implicites; ce qui nous aide à bien traduire les idées de l'auteur. Parfois, nous ajoutons des mots pour rendre les gestes plus claires chez le nouveau lecteur. Par exemple, en traduisant l'énoncé elle secoue la tête, nous ajoutons l'énoncé arabe (علامة الرفض) . Cela n'existe pas dans le texte source mais il est compris implicitement à partir du contexte. D'après le contexte, nous pouvons dire : فهزت رأسها علامة الرفض/ بالموافقة تأكيدا. C'est presque pareil pour les énoncés répétés je hoche la tête, je réponds de la tête, il hausse les épaules qui se traduisent selon leur contexte. Notre intervention consiste à rendre clair tout sens implicite provoqué par ce langage gestuel. Si ces gestes restent implicites dans le texte d'arrivée, ils risqueraient d'échapper au destinataire de la traduction. Il est donc nécessaire de les lui présenter de façon suffisamment explicite pour qu'il comprenne le message comme le lecteur du texte source.

Tout au long du passage du Français en Arabe, nous nous efforçons de reproduire dans le texte cible les mêmes gestes répétés. Ces gestes traduisent les émotions partagées par les locuteurs. En effet, la construction d'un lexique précoce relatif aux émotions, semble tellement importante que si l'on est transporté de joie, fou de rage, envahi de tristesse ou rongé par la douleur, c'est dans la langue maternelle qu'on cherche à s'exprimer, souvent d'une manière spontanée et involontaire. La langue maternelle et les émotions sont intimement liées : c'est à partir des outils 
linguistiques de sa propre langue que l'individu construit l'expression de ses émotions. Et c'est par l'intermédiaire de cette langue qu'il préfère véhiculer et communiquer ses émotions et ses sentiments.

Dans le texte source, l'auteur lui-même ne fait que traduire ses impressions et ses émotions en langue étrangère. Il nous décrit les émotions et les sentiments de ces personnages par les gestes, les mimiques et les signes non verbaux. Nous essayons ici d'interpréter ce langage non-verbal pour reproduire les mêmes effets voulus par l'auteur chez le nouveau lecteur. Aux termes de notre travail, nous nous servons des gestes, des actions verbales et non-verbales, des non-dits et d'autant de manifestations de l'attitude des locuteurs pour mettre en relief l'effet que l'auteur voudrait produire auprès de son lecteur.

En retour à la citation ci-dessus, nous pourrions dire qu'outre les gestes et les mimiques, elle nous plonge dans un désarroi complet. C'est le malheur d'un homme qui participe au régime. Cet homme perd à la fois sa dignité et sa liberté dans son pays. Il est obligé d'être humilié et prisonnier dans son pays. C'est un être pris dans un piège aux motifs obscurs.

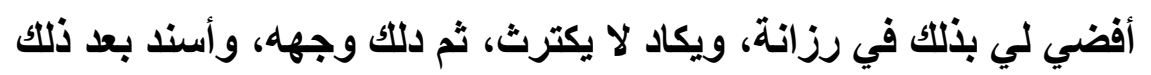

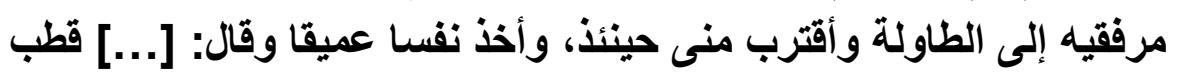

$$
\begin{aligned}
& \text { حاجبيه، وأضاف: } \\
& \text { - لقد بدا جلياً لها مثلما بدا لي: إما الخضوع أو الموت؛ فأن تتكلم } \\
& \text { أو تجادل أو تبدى احتجاجك يكاد ذلك لا يجدي نفعا. }
\end{aligned}
$$


Les signaux doivent être interprétés en fonction du contexte. La signification d'un geste dépendrait en fait de la situation, de l'émetteur, du récepteur, de la culture, de la religion. Quand nous traduisons l'énoncé "il frappe sur le volant et jure plusieurs fois" ${ }^{11}$, il faut penser ici au regret et à la douleur qu'éprouve le locuteur. Ajoutons le mot arabe qui nous paraît implicite dans l'énoncé source pour exprimer le sentiment du locuteur en disant وضرب عجلة Quant à l'énoncé jure plusieurs fois, nous l'avons traduit par وأقسم عدة مرات, sans rien ajouter.

Tout au long du texte, l'auteur essaie d'observer et de maîtriser le mouvement articulatoire et intonatif de ses personnages. Il ne laisse pas échapper leurs répliques sans ajouter un commentaire sur l'articulation ou insister sur le ton de certains énoncés. Cela vient peut-être de la difficulté à deviner le ton puisque c'est une ouvre écrite, une manière de faciliter pour le lecteur, la tâche d'interprétation. Ce jeu articulatoire que le narrateur effectue, reflète bel et bien ses propos. De plus, le narrateur joue parfois sur le ton en simulant son indifférence pour laisser entendre un message qu'il trouve dur à faire passer directement :

«"Ca va ?" lui adresse gentiment Bamezon.

La jeune femme répond d'une voix timide :

"Très bien, monsieur."

1 ) Ibid. P.49 
Puis il ajoute soudain gaiment, haussant la voix afin de couvrir les cris de la chanteuse.

"Madame Nadine aussi est là."

Elle se retourne, et indique la zone sous l'arbre. "Là-bas."

Bamezon hoche la tête.

"Vous pouvez l'informer de ma présence."

Il se penche ensuite vers moi :

"Elle va venir s'asseoir avec nous. Ça ne t'ennuie pas?"

Cela me fait même assez plaisir de les voir ensemble». ${ }^{1}$

Cette longue citation ci-dessus met l'accent sur les différents aspects du langage corporel : gestes, mouvements intonatifs, mimiques et d'autres signes non verbaux. Ces signes non verbaux jouent un rôle prépondérant dans la coordination de la conversation humaine et ils ont un impact plus important sur l'auditeur que le contenu textuel du message exprimé. Ils doivent être interprétés en fonction du contexte. La signification d'un geste dépend en premier ordre du contexte. Nous avons transféré ces gestes répétés tout au long du texte par des gestes semblables. Ceux-ci expriment le plus souvent les mêmes effets voulus par l'auteur. Cela ne se passe sans l'ajout de quelques mots qui rend explicite ce qui est essentiel pour la compréhension du lecteur de la traduction. Comparons cette traduction à l'extrait précédent :

$$
\text { فخاطبها باميزون بلطافة قائلا: كيف حالك؟ }
$$

1 ) Ibid. P.65 


$$
\begin{aligned}
& \text { - بخيز با سبدي. } \\
& \text { ثم أضافت فجأة وقد ارتسمت على مُحياها البشاشة، وقالت بصوث مرثفع } \\
& \text { ليطغى على طنين المغتية: } \\
& \text { - السيدة نادين تجلس هنائ. } \\
& \text { واستدارث على عاقبيها وقصدث الموضع أسفل الشجرة، وقالت: } \\
& \text { - } \\
& \text { فأجاب باميزون بهز رأسه متفهما، وقال: } \\
& \text { - بإمكانك أن تخبرينها بقدومي. } \\
& \text { ثم مال ناحيتي وقال: } \\
& \text { - توشك على الجلوس معنا، أما يزعجك هذا؟ } \\
& \text { وكم ينشرح صدري عندما أراهما معا. }
\end{aligned}
$$

Ainsi, un geste quelconque peut nous informer d'une façon implicite sur ce qui reste non-dit verbalement. En outre, l'auteur emploie quelques expressions telles que $\boldsymbol{t u}$ me comprends, il fait un geste, il hoche la tête, pousse un soupir, il plisse le front, il me regarde, me fixe dans les yeux, il se tait, il se penche, etc. Le narrateur répète à plusieurs fois ces termes comme pour retenir l'expression fuyante et la mettre à la portée de son destinataire, ce qui montre son attachement à ne communiquer qu'implicitement une vérité ou une opinion. Observons l'exemple suivant : Il se penche vers moi. «Un moraliste ; voilà ce qu'il était. De la tête aux pieds. Il n'avait rien à faire au milieu de ces hyènes. Tu me comprends ?» 1

1) Ibid. P. 131 
Cette citation marque une idée cherchée à la fois par le narrateur et par le lecteur dès le début du texte source. C'est de savoir comment le conseiller du président est tué. L'Allemand Uwe nous fait découvrir indirectement la manière dont Bamezon est mort. Ce dernier n'est qu'un homme de lettres et n'a pas d'affaire à la politique. Il se trompe de poste. Il se trouve enfin au milieu de ceux qui exercent impitoyablement le pouvoir. Lors de la traduction, nous devons compléter les énoncés elliptiques pour transposer complètement les idées sources en des énoncés accessibles :

$$
\text { ومال ناحيتي، وأضاف: }
$$

Du reste, le texte source nous révèle des réalités très importantes. Le nom propre de Dahomey, appelé aussi Abomey, nous fait penser non seulement aux grands palais mais aussi à l'histoire de l'esclavage. Le commerce esclavagiste y a été pratiqué longtemps. En décrivant la réalité cruelle de son pays, le narrateur pose des questions très importantes tout au long du texte. Ces questions qui traduisent le pessimisme et l'angoisse de l'auteur resteront sans réponse :

«Ce sable fin a vu s'échanger des captifs pendant des siècles. Mais ces femmes usées avant l'âge par le travail physique ne sont-elles pas des esclaves? Et ces hommes, la, couchés dans la poussière, ont-ils une existence meilleure 
que leurs ancêtres déportés? [...] je suis pris d'une émotion indéfinie-une sorte de crainte et d'aversion mélangées pour le monde et les hommes- qui me fait battre le caur très fort. " ${ }^{1}$

Cette citation met en évidence la vie cruelle des pauvres africains. Le lieu témoigne de la condition des esclaves et de la capture des prisonniers aux grands royaumes du Togo. L'auteur fournit ici des idées bien réelles. Celles-ci existent dans toutes les têtes des émigrés africains. Il écrit librement ce que certains n'osent pas dire. Cependant, nous avons pour but de faire passer un message, de créer un texte cible agréable à lire tout en se servant des idées explicites et implicites. Pour le passage cidessus, nous avons introduit dans le texte cible les énoncés encadrés qui traduisent à leur tour ce qui n'est pas directement exprimé :

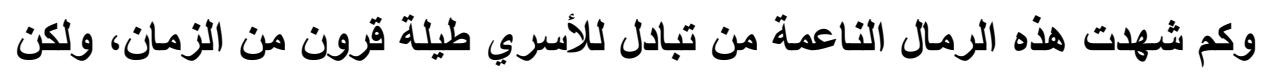

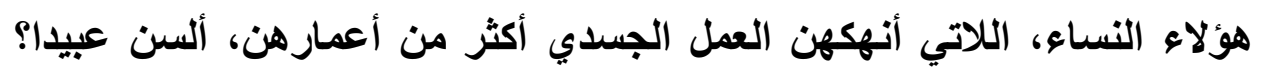

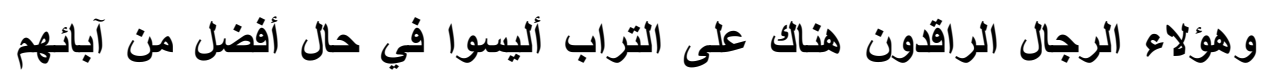

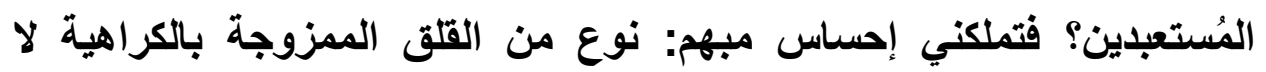

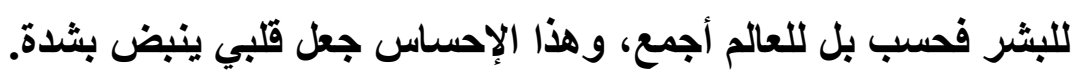

C'est au cours de notre travail que nous avons mis sous les yeux la compréhension du nouveau lecteur, en restant relativement fidèle au texte source. Il nous semble nécessaire de présenter au destinataire de la traduction des significations laissées implicites de façon explicite pour que

1 ) Ibid. P. 123-124 
ce dernier puisse saisir le message transmis par le texte source. Nous voudrions ici finir par la parole de Seleskivitch : «Pour dire clairement ce qu'il entend, chacun choisit l'explicite adapté à la fois à l'idée qui lui est particulière et aux connaissances de l'autre. À ce premier rapport explicite/implicite se superpose le rapport explicite/implicite créé par chacune des langues et différent dans chacune d'elles. " ${ }^{1}$

Ainsi, nous avons abordé, dans les pages ci-dessus, un problème d'ordre sémantique, la traduction de l'implicite dans ténèbres à midi de Théo Ananissoh. Comme nous l'avons dit, la traduction est le résultat d'un effort linguistique très complexe commandé par le souci de transmettre un contenu d'idées. Tous les aspects et toutes les phases de ce processus sont donc subordonnés à ce souci qui est essentiellement une préoccupation sémantique. La sémantique est alors au cour de cette opération.

En conclusion à cette recherche, nous pouvons dire que l'interprétation de l'implicite constitue un problème indéniable lors de la traduction. L'implicite est alors l'un des problèmes sémantiques de la traduction. Ainsi, il faut avouer que les notions de perception, de compréhension et de mémorisation sont des outils assez importants dans l'objectif d'identifier la valeur implicite dans un roman à traduire. Par conséquent, dans l'optique de la traduction de cette ouvre, le traducteur doit la lire avec patience et

\footnotetext{
$\left.{ }^{1}\right)$ Seleskovitch D. \& Lederer M. : Interpréter pour traduire, Paris, Didier Erudition, 3ème édition - revue et corrigée, 1993, p. 183.
} 
discernement, afin de déceler ce que cache l'implicite, ce que les mots révèlent au-delà de leur message premier. Une bonne traduction implique alors de comprendre le texte à traduire pour le transposer aussi finement que possible dans une langue cible.

\section{Bibliographie}

I- Corpus :

- ANANISSOH Théo : Ténèbres à midi, Gallimard, Paris, 2010, 138 p.

\section{II- Guvres :}

- ALBIR Amparo Hurtado : La Notion de fidélité en traduction, Paris, Didier- Erudition, 1990.

- BENVENISTE Emile, Problèmes de linguistique générale, Paris, Gallimard, 1976.

- BRAVO Nicole Fernandez : Lire entre les lignes : l'implicite et le non-dit, Asnières, PIA, coll.«Publications de l'Institut d'Allemand d'Asnières», 2003.

- CATHERINE Kerbrat-Orecchioni: L'implicite, Paris, Armand Colin, 1986

- CATHERINE Kerbrat-Orecchioni : L'énonciation : de la subjectivité dans le langage, Paris : A. Colin, 2002.

- DELISLE Jean : L'analyse du discours comme méthode de traduction, Canada, Presse Universitaire d'Ottawa, 1982.

- KLEIBER Georges : Problèmes de sémantique. La polysémie en question, Paris, Presses universitaires du Septentrion, 1999. 
- LALAOUI-CHIAL Fatima Zohra : Guide de sémiotique appliquée, Oran, OPU, 2008.

- LEDERER Marianne, et SELESKOVITCH Danica : Interpréter pour traduire, 4ème édition revue et corrigée, Paris, Didier Erudition, 2001.

- MOUNIN Georges : Les Problèmes théoriques de la traduction, Paris, Gallimard, 1963.

- SELESKOVITCH Danica et LEDERER Marianne : Interpréter pour traduire, Paris, Didier- Erudition, 1993.

- SENGHOR Léopold Sédar. Ethiopiques. Paris : Editions du Seuil, 1956

- VINAY Jean-Paul \& DARBELNET Jean : Stylistique comparée du français et de l'anglais. Méthode de traduction, Paris, Didier, 1977.

YUSTE FRIAS José : Au seuil de la traduction : la paratraduction, in Naaijkens, New York, Oxford, 2010.

\section{III- Articles :}

- BARTHES Roland : Introduction à l'analyse structurale des récits, in Communications, $\mathrm{n}^{\circ} 8$, Paris, Seuil, École pratique des hautes études 6e section, 1966, pp. 1-27.

- EL BADAOUI AHMED Manal: Traduction de quelques faits culturels du français vers l'arabe : retour de l'original à son point d'origine, traduction, terminologie, rédaction, (TTR) vol. 25, $\mathrm{n}^{\circ} 1,2012$.

- HONG VAN Dinh : La théorie du sens et la traduction des facteurs culturels, Synergies Pays riverains du Mékong, $\mathbf{n}^{\circ}$ 1,2010, pp. 141-171. 